\title{
Aspectos fitossociológicos e indicadores da qualidade do solo em sistemas agroflorestais
}

\author{
Lucas Henrique Vieira LENCI ${ }^{1}$, Emanuel Fernando Maia de SOUZA ${ }^{2}$, \\ Adriano Reis Prazeres MASCARENHAS ${ }^{2}$, Antonio de Arruda TSUKAMOTO FILHO ${ }^{1}$, \\ Gilderlon dos Santos SOARES ${ }^{2}$
}

\author{
${ }^{1}$ Programa de Pós-Graduação em Ciências Florestais e Ambientais, Universidade Federal de Mato Grosso, Cuiabá, \\ Mato Grosso, Brasil. \\ ${ }^{2}$ Departamento de Engenharia Florestal, Universidade Federal de Rondônia, Rolim de Moura, Rondônia, Brasil. \\ E-mail: lucashenriquevl@unir.br
}

Recebido em março/2018; Aceito em agosto/2018.

\begin{abstract}
RESUMO: Objetivou-se avaliar os atributos florísticos e fitossociológicos, os atributos físicos do solo e os estoques de matéria orgânica e carbono orgânico do solo de cinco sistemas agroflorestais (SAFs) da Região Central de Rondônia. Para isso, realizou-se o levantamento florístico para determinação da área basal, riqueza, diversidade e uniformidade das espécies. No processo de amostragem do solo, dividiu-se cada um dos sistemas em quatro quadrantes e coletou-se amostras indeformadas nas profundidades de $0 \mathrm{~cm}$ a $20 \mathrm{~cm}$ e $20 \mathrm{~cm}$ a $40 \mathrm{~cm}$ para determinação da densidade do solo, macroporosidade, microporosidade e porosidade total; e amostras deformadas nas profundidades de $0 \mathrm{~cm}$ a $5 \mathrm{~cm}, 5 \mathrm{~cm}$ a $10 \mathrm{~cm}, 10 \mathrm{~cm}$ a $20 \mathrm{~cm}$ e $20 \mathrm{~cm}$ a $40 \mathrm{~cm}$ para determinação dos estoques de matéria orgânica e carbono orgânico do solo. Os resultados foram submetidos à correlação de Pearson para verificar interações entre os parâmetros avaliados. Os atributos florísticos e fitossociológicos variaram consideravelmente entre os sistemas agroflorestais e, de modo geral, os diferentes arranjos de SAFs apresentaram atributos físicos próximos ou abaixo dos valores considerados críticos para crescimento radicular. Além disso, notou-se que os SAFs mais adensados e com estrutura do solo favorável ao desenvolvimento das plantas favoreceram maiores quantidades de matéria orgânica e carbono orgânico do solo.
\end{abstract}

Palavras-chave: agroecossistemas, física do solo, estoque de carbono, desenvolvimento rural sustentável.

\section{Phytosociological aspects and soil quality indicators in agroforestry systems}

\begin{abstract}
This study aimed to analyze floristic and phytosociological attributes, soil physical attributes, and organic matter and soil organic carbon stocks in five agroforestry systems (AFS) in the Central Region of Rondônia. Floristic surveys were carried out to determine basal area, richness, diversity and uniformity of the species. In the soil sampling process, each of the systems were divided into four quadrants, and undisturbed soil core samples were collected at 0-20 and 20-40 cm depths to determine soil bulk density, macroporosity, microporosity and total porosity; disturbed soil core samples were collected at 0-5, 5-10, 10-20 and 20-40 cm depths to determine soil organic matter and carbon organic stocks. Pearson correlation was applied to evaluate significant interactions among the results. Floristic and phytosociological attributes varied considerably between agroforestry systems. In general, the agroecosystems showed soil physical attributes close to or below the values considered critical for root growth. Favorable soil structure and denser arrangements resulted in higher amounts of organic matter and organic carbon in the soil.
\end{abstract}

Keywords: agroecosystems, soil physics, carbon stocks, sustainable rural development.

\section{INTRODUÇÃO}

Como resultado das últimas décadas de desenvolvimento socioeconômico, a região Amazônica tem registrado intensa pressão sobre seus recursos naturais. Estimativas apontam que cerca de 85 milhões de hectares da Floresta Amazônica foram desmatados desde a década de 1970, devido, principalmente, a conversão de florestas em pastagens (MALHI et al., 2008). Atualmente os fatores que mais contribuem para 0 desmatamento, degradação ambiental e perda da biodiversidade são os programas de desenvolvimento regional, com destaque para os projetos de usinas hidrelétricas e as rodovias que ligam a Amazônia ao Pacífico (FEARNSIDE; GRAÇA, 2009), além do intenso crescimento populacional e da contínua expansão das fronteiras amazônicas (CARR et al., 2009).
Considerando esta realidade de uso destrutivo da biodiversidade amazônica, faz-se necessário promover e adotar sistemas de produção sustentáveis que associem a questão econômica com a conservação dos recursos naturais. Um exemplo são sistemas agroflorestais (SAFs), que compreendem o cultivo simultâneo de árvores, espécies agrícolas e/ou animais seguindo determinado arranjo espacial e temporal (ALTIERI, 2012). Devido sua composição e estrutura, estes agroecossistemas são funcionalmente mais complexos e com maior eficiência de captura e utilização dos recursos, tais com nutrientes, luz e água, do que os sistemas convencionais de cultivo (SILVA et al., 2011a).

Portanto, os sistemas agroflorestais configuram-se como alternativas para promoção do desenvolvimento rural sustentável, pois podem apresentar resultados positivos em 
relação à proteção ambiental, ao crescimento econômico e às melhorias das condições sociais. Esta proteção ambiental possibilitada pelos sistemas agroflorestais decorre do fato de sua estrutura, composição e função se assemelhar dos ecossistemas naturais, realizando processos ecológicos complexos e proporcionando diversos serviços ambientais.

Devido ao intenso aporte de material vegetal que promove a cobertura do solo, a manutenção da matéria orgânica e a ciclagem de nutrientes, uma das possíveis contribuições dos sistemas agroflorestais é a melhoria dos atributos edáficos (PEZARICO et al., 2013). Estes agroecossistemas apresentam atributos físicos do solo, como densidade, porosidade, tamanho das partículas, infiltração de água e resistência à penetração, que favorecem o crescimento e desenvolvimento das plantas, aumentando a produtividade e garantindo a sustentabilidade do sistema produtivo.

Adicionalmente, outro importante elemento das agroflorestas é a intensa dinâmica do carbono orgânico do solo, haja vista que pesquisas recentes têm apontado que os SAFs conservam, e até mesmo aumentam, os estoques de carbono do solo, em comparação com outros sistemas menos complexos, como pastagem e plantio convencional (LIMA et al., 2011). Em virtude dessa complexidade de estrutura e composição florística, os sistemas agroflorestais produzem maior quantidade de biomassa, o que favorece a cobertura do solo, o acúmulo de carbono orgânico, a manutenção da fertilidade do solo e a redução da emissão de gases de efeito estufa (GAMA-RODRIGUES et al., 2008; PARRON et al., 2015).

Dessa forma, admitindo-se que os atributos físicos e o carbono orgânico do solo são considerados eficientes indicadores ambientais em sistemas produtivos (SILVA et al., 2011a), o levantamento dessas informações nos diferentes sistemas de uso da terra existentes na Amazônia é de grande relevância na determinação das melhores práticas e técnicas de manejo.

Diante desses aspectos, objetivou-se com este trabalho, associar os atributos físicos e estoques de carbono do solo com os atributos florísticos e fitossociológicos de diferentes sistemas agroflorestais da Região Central de Rondônia.

\section{MATERIAL E MÉTODOS}

$\mathrm{O}$ estudo foi realizado em cinco sistemas agroflorestais da Região Central do estado de Rondônia, sendo três sistemas (SAF 1, SAF 2 e SAF 3) no município de Rolim de Moura RO e dois sistemas (SAF 4 e SAF 5) no município de Cacoal - RO (Figura 1).

O clima da região é considerado do tipo Am segundo a classificação climática de Köppen-Geiger, caracterizada por temperatura média anual de $25^{\circ} \mathrm{C}$, altitude entre 200 e $400 \mathrm{~m}$ e precipitação total anual entre 2300 a $2500 \mathrm{~mm}$ (ALVARES et al., 2013). O solo predominante na região de Rolim de Moura é Cambissolo Háplico Ta Eutrófico (CXve) e na região de Cacoal é Argissolo Vermelho-Amarelo Eutrófico (PVAe) (SANTOS et al., 2013).

\subsection{Caracterização dos sistemas agroflorestais}

Os SAFs estudados em Rolim de Moura estão em propriedades rurais diferentes, entretanto todos foram implantados há 30 anos e, periodicamente, são submetidos a limpeza mecanizada com máquinas de pequeno porte.
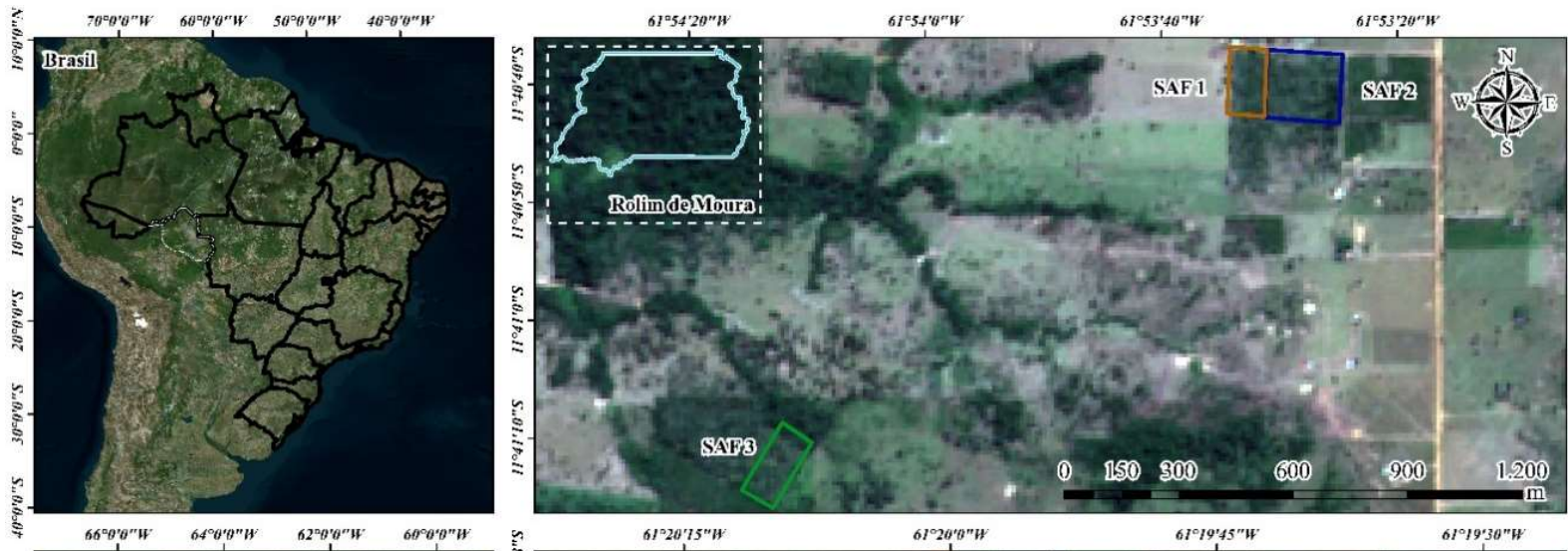

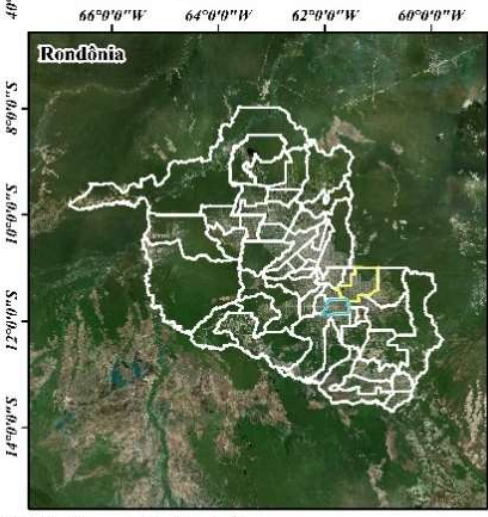

Satélite/Sensor: Sentinel - 2 Rotas/Cenas: 20L.PN. Data do Imageamento: 27 de junho de 2017.

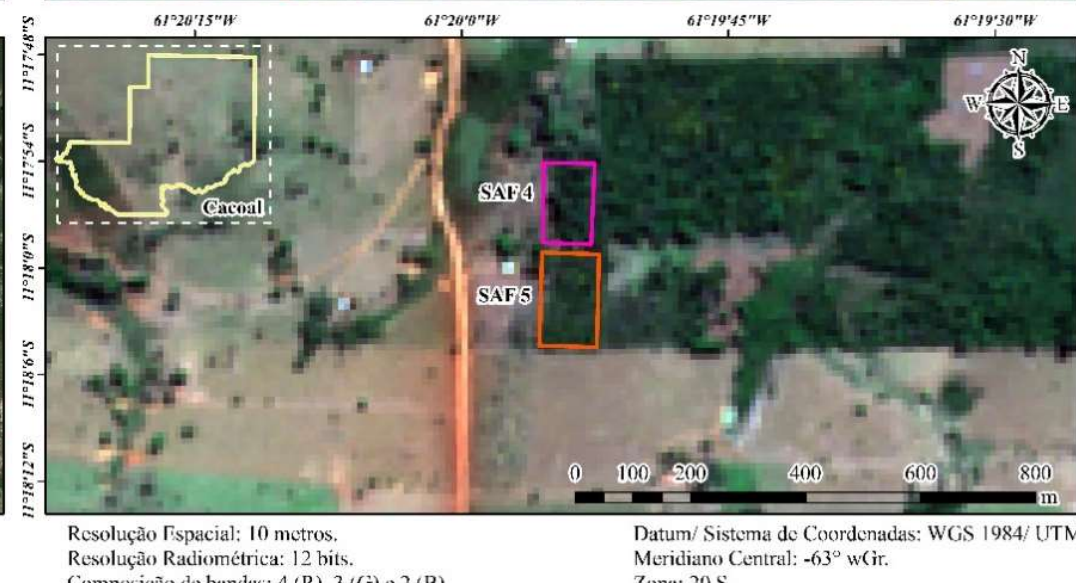

Resoluçâo Radiométrica: 12 bits.

Composicão de bandas: 4 (R), 3 (G) e 2 (B).
Meridiano Central: $-63^{\circ}$ w(ir

7.ona: $20 \mathrm{~S}$.

Figura 1. Mapa de localização dos sistemas agroflorestais em estudo nos municípios de Rolim de Moura e Cacoal, na Região Central do estado de Rondônia.

Figure 1. Location map of the agroforestry systems under study in the municipalities of Rolim de Moura and Cacoal, in the Central Region of the state of Rondonia. 
O SAF 1 tem área de 1,41 ha e composição florística baseada no consórcio de seringueira (Hevea brasiliensis Müll.Arg.) com cupuaçuzeiro (Theobroma grandiflorum K. Schum), além da presença, em menor densidade, das espécies cacaueiro (Theobroma cacao L.), castanheira (Bertholletia excelsa Bonpl.), cedro (Cedrela fissilis Vell.) e palmeiras. O solo nesse sistema apresenta coloração escura e a textura argilosa.

Já o SAF 2 apresenta área de 3,28 ha e a composição florística consiste no predomínio de seringueira, mas com significativa presença de cupuçuzeiro, bandarra (Schizolobium amazonicum Huber ex Ducke), timbaúva (Enterolobium contortisiliquum (Vell.) Morong), embaúba (Cecropia sp.), oiticica (Licania rigida Benth.) e de açaizeiros (Euterpe sp.) em estágio inicial de desenvolvimento. Nesse SAF o solo tem coloração amarelo escuro e textura visualmente mais arenosa do que o SAF 1.

E em relação ao SAF 3, a área de cultivo corresponde a 10,5 ha e tem a composição florística constituída pelo consórcio de seringueira com cupuçuzeiro, com presença de outras espécies como embaúba, escova-de-macaco (Mayna grandifolia (H.Karst.) Warb.), louro (Cordia sp.) e mandiocão (Schefflera morototoni (Aubl.) Maguire et al.), em menor densidade. O solo da área é nitidamente o mais arenoso dentre os sistemas analisados e apresenta coloração cinza claro.

Os sistemas agroflorestais da região de Cacoal, SAF 4 e SAF 5, estão localizados na mesma propriedade rural e são oriundos do cultivo remanescente de cafeeiros (Coffea sp.) com espécies florestais tais como bandarra, embaúba, ipê (Tabebuia sp.), paineira (Ceiba sp.) e baginha (Samanea tubulosa Benth.). O SAF 4 tem 0,90 ha e foi implantado há 39 anos, enquanto o SAF 5 tem 0,35 ha e foi implantado há 6 anos. Ambos sistemas apresentam solos com coloração vermelho escura e textura nitidamente argilosa.

A classificação de textura e cor dos solos, dos respectivos SAFs, foi baseada na aplicação da carta de Munsell e na metodologia de identificação tátil e visual preconizada na NBR 6484 (ABNT, 2001).

\subsection{Levantamento florístico e fitossociológico}

Para obter as informações da vegetação dos sistemas agroflorestais, realizou-se o levantamento com mensuração e identificação ao menor nível taxonômico de todos os indivíduos com diâmetro a altura do peito (DAP) maior ou igual a $5 \mathrm{~cm}$. Para os SAFs com tamanho inferior a 1 ha (SAF 4 e SAF 5) realizou-se o censo e para os SAFs com área superior a 1 ha (SAF 1, SAF 2 e SAF 3), aplicou-se procedimento de amostragem em parcelas de 40 por $50 \mathrm{~m}$, totalizando 1 ha amostrado.

Os dados foram processados no software FITOPAC 2.1.2 para cálculo dos parâmetros fitossociológicos: área basal $(\mathrm{G})$ (Equação 1), riqueza de espécies (S), Índice de Diversidade de Shannon (H') (Equação 2) e uniformidade das espécies, por meio do Índice de Equabilidade de Pielou (J') (Equação 3 e Equação 4).

$$
\begin{gathered}
G=\sum_{n=1}\left(\frac{\pi \times D A P_{n}^{2}}{4}\right) \\
H^{\prime}=-\sum_{i=1}^{S} \frac{n_{i}}{N} \ln \frac{n_{i}}{N}
\end{gathered}
$$

$$
\begin{gathered}
J^{\prime}=\frac{H^{\prime}}{H_{\max }} \\
H_{\max }=\ln (S)
\end{gathered}
$$

em que: $\mathrm{G}=$ área basal $\left(\mathrm{m}^{2} \mathrm{ha}^{-1}\right)$; DAP = diâmetro a altura do peito do indivíduo $\mathrm{n} ; \mathrm{H}^{\prime}=$ índice de diversidade de Shannon; $\mathrm{n}_{\mathrm{i}}=$ número de indivíduos amostrados para a i-ésima espécie; $\mathrm{N}$ = número total de indivíduos amostrados; $\mathrm{J}^{\prime}=$ índice de equabilidade de Pielou; $\mathrm{S}=$ riqueza de espécies.

\subsection{Amostragem do solo}

Cada um dos SAFs avaliados foi subdividido em quatro quadrantes, que constituíram as unidades amostrais de cada sistema. Para as análises dos atributos físicos do solo, realizouse em cada quadrante a abertura de uma trincheira com dimensões de $40 \mathrm{~cm} \times 70 \mathrm{~cm} \times 40 \mathrm{~cm}$ (largura $\times$ comprimento $\times$ profundidade). Em seguida, realizou-se a coleta de duas amostras indeformadas de solo, com cilindros metálicos de $4 \mathrm{~cm}$ de altura e $6 \mathrm{~cm}$ de diâmetro interno, nas profundidades de $0 \mathrm{~cm}$ a $20 \mathrm{~cm}$ e $20 \mathrm{~cm}$ a $40 \mathrm{~cm}$.

Para as análises do teor e estoque de carbono do solo, realizou-se a coleta de cinco amostras deformadas nas profundidades $0 \mathrm{~cm}$ a $5 \mathrm{~cm}, 5 \mathrm{~cm}$ a $10 \mathrm{~cm}, 10 \mathrm{~cm}$ a $20 \mathrm{~cm}$ e $20 \mathrm{~cm}$ a $40 \mathrm{~cm}$, em cada unidade amostral, por meio de trado do tipo holandês. Essas amostras foram então homogeneizadas para formar uma amostra composta por quadrante.

\subsection{Atributos físicos do solo}

As amostras coletadas foram encaminhadas para o Laboratório de Solos da Universidade Federal de Rondônia, Campus Rolim de Moura, onde foi executada a metodologia preconizada por Donagema et al. (2011) para determinação dos atributos físicos: densidade do solo (Ds) (Equação 5), microporosidade (Mip) (Equação 6), macroporosidade (Map) (Equação 7) e porosidade total (Pt) (Equação 8).

$$
\begin{gathered}
\text { Ds }=\frac{\mathrm{m}}{\mathrm{v}} \\
\text { Mip }=\left(\frac{\mathrm{a}-\mathrm{b}}{\mathrm{c}}\right) \\
\text { Map }=\text { VS }- \text { Mip } \\
\mathrm{Pt}=\text { Mip }+ \text { Map }
\end{gathered}
$$

em que: Ds = densidade do solo, em $\mathrm{kg} \mathrm{dm}^{-3} ; \mathrm{m}=$ massa da amostra seca a $105{ }^{\circ} \mathrm{C}$, em $\mathrm{mg} ; \mathrm{v}=$ volume do cilindro, em $\mathrm{m}^{3}$; Mip = microporosidade, em $\mathrm{m}^{3} \mathrm{~m}^{-3}$; $\mathrm{a}=$ massa de amostra após ser submetida a uma tensão de $60 \mathrm{~cm}$ de coluna de água, em kg; $b=$ massa da amostra seca a $105^{\circ} \mathrm{C}$, em kg; c = volume do cilindro, em $\mathrm{m}^{3}$; Map $=$ macroporosidade, $\mathrm{em} \mathrm{m}^{3} \mathrm{~m}^{-3} ; \mathrm{VS}=$ volume de saturação, $\mathrm{em} \mathrm{m}^{3} \mathrm{~m}^{-}$ 3; $\mathrm{Pt}=$ porosidade total, $\mathrm{em} \mathrm{m}^{3} \mathrm{~m}^{-3}$.

2.5. Teor de carbono, matéria orgânica e estoque de carbono do solo

Para a determinação dos teores de carbono do solo, preparou-se a amostra de solo deformada por meio de secagem, destorroamento e peneiramento em malha de $2 \mathrm{~mm}$, para obtenção de terra fina seca ao ar (TFSA). Após essa etapa, realizou-se a trituração de, aproximadamente, $2 \mathrm{~g}$ de TFSA em almofariz e peneiramento em malha de $0,25 \mathrm{~mm}$, a fim de se obter o material em aspecto de pó (DONAGEMA et al., 2011). 
Em seguida, foi realizada a determinação do teor de carbono orgânico do solo para cada uma das profundidades amostradas, por meio da metodologia proposta por Rodrigues et al. (2016) para as condições amazônicas. Este método consiste na adição sequencial, em recipiente erlermeyer de 125 $\mathrm{mL}$, de $0,5 \mathrm{~g}$ do solo em pó (aferido em balança de precisão de 0,001), $10 \mathrm{~mL}$ de dicromato de potássio a $0,166 \mathrm{M}, 10 \mathrm{~mL}$ de ácido sulfúrico concentrado, $3 \mathrm{~mL}$ de ácido ortofosfórico concentrado e $47 \mathrm{~mL}$ de água destilada ou deionizada com mais 10 gotas do indicador difenilamina $\left(10 \mathrm{~g} \mathrm{~L}^{-1}\right)$.

Após o resfriamento da solução preparada, realizou-se, com o auxílio de bureta digital e agitador magnético, a titulação das amostras com sulfato ferroso amoniacal (0,5 M) até a transição da cor da mistura. Após obter o valor do volume gasto da solução, foi possível realizar o cálculo do teor de carbono orgânico do solo (Equação 9) para cada uma das amostras.

Os teores foram determinados para as camadas de $0 \mathrm{~cm}$ a $20 \mathrm{~cm}$ (média das amostras das profundidades $0 \mathrm{~cm}$ a $5 \mathrm{~cm}, 5 \mathrm{~cm}$ a $10 \mathrm{~cm}$ e $10 \mathrm{~cm}$ a $20 \mathrm{~cm}$ ) e $20 \mathrm{~cm}$ a $40 \mathrm{~cm}$. Com os valores de teor de carbono, aplicou-se o fator de 1,724 para calcular a matéria orgânica do solo (Equação 10). Por último, foi possível realizar os cálculos de estoque total de carbono orgânico do solo (Equação 11).

$$
\begin{array}{cc}
\mathrm{C}=\frac{(\mathrm{BR}-\mathrm{AM}) \times 3,9 \times\left(\frac{10}{\mathrm{FT}}\right)}{\mathrm{MA}} & (\text { Equação 9) } \\
\text { MOS }=\mathrm{C} \times 1,724 & (\text { Equação 10) } \\
\text { Est COS }=\frac{(\mathrm{C} \times \mathrm{Ds} \times \mathrm{e})}{10} & (\text { Equação 11) }
\end{array}
$$

em que: $\mathrm{C}=$ carbono total da amostra, em $\mathrm{g} \mathrm{kg}^{-1} ; \mathrm{BR}=$ volume de sulfato ferroso amoniacal utilizado para titular prova em branco, em $\mathrm{mL} ; \mathrm{AM}=$ volume de sulfato ferroso amoniacal utilizado para titular a amostra, em $\mathrm{mL} ; \mathrm{FT}$ = fator de correção para dicromato de potássio; $\mathrm{MA}=$ massa de solo utilizada para determinação de $\mathrm{C}$, em g; MOS = matéria orgânica do solo, em $\mathrm{Mg} \mathrm{ha}^{-1}$; Est COS = estoque de carbono orgânico em determinada profundidade, em $\mathrm{Mg} \mathrm{ha}^{-1}$; Ds = densidade do solo da profundidade, em $\mathrm{g} \mathrm{cm}^{-3}$; e $=$ espessura da camada considerada, em $\mathrm{cm}$.

\subsection{Processamento e análise dos resultados}

O conjunto de dados foi submetido ao teste de normalidade de Shapiro-Wilk, a 5\% de significância. Posteriormente, os atributos físicos do solo, atributos florísticos e os teores de carbono, matéria orgânica e estoques de carbono do solo foram avaliados por meio de estatística descritiva indicando-se valores médios, desvio padrão e coeficiente de variação.

Além disso, todos os parâmetros obtidos foram submetidos à análise de correlação de Pearson a 10\% de probabilidade.

\section{RESULTADOS}

Os valores de área basal $(\mathrm{G})$, riqueza de espécies (S), Índice de Diversidade de Shannon (H') e Índice de Equabilidade de Pielou ( $\left.\mathrm{J}^{\prime}\right)$ estão apresentados na Tabela 1. Conforme previsto, os resultados expressos referentes aos atributos florísticos e fitossociológicos variaram consideravelmente entre os cinco sistemas agroflorestais analisados.

Em relação à área basal, por exemplo, o SAF 1 apresentou o maior valor $\left(23,52 \mathrm{~m}^{2} \mathrm{ha}^{-1}\right)$ e o SAF 2 o menor valor $(6,55$ $\left.\mathrm{m}^{2} \mathrm{ha}^{-1}\right)$. Isto acontece em virtude da diferença de espaçamento entre as árvores, sendo que geralmente arranjos mais adensados, como os SAF 1 e SAF 3, resultam em maiores valores de área basal.

Tabela 1. Atributos florísticos e fitossociológicos de diferentes sistemas agroflorestais da Região Central do Estado de Rondônia.

Table 1. Floristic and phytosociological attributes of different agroforestry systems in the Central Region of Rondonia.

\begin{tabular}{ccccc}
\hline \multirow{2}{*}{ Agroecossistemas } & \multicolumn{4}{c}{ Atributos florísticos } \\
\cline { 2 - 5 } & $\begin{array}{c}\mathrm{G} \\
\left(\mathrm{m}^{2} \mathrm{ha}^{-1}\right)\end{array}$ & $\begin{array}{c}\mathrm{S} \\
\text { (espécies) }\end{array}$ & $\mathrm{H}^{\prime}$ & $\mathrm{J}^{\prime}$ \\
\hline SAF 1 & 23,52 & 15 & 0,765 & 0,283 \\
SAF 2 & 6,55 & 28 & 0,598 & 0,179 \\
SAF 3 & 18,22 & 36 & 1,111 & 0,308 \\
SAF 4 & 15,68 & 23 & 2,652 & 0,846 \\
SAF 5 & 16,71 & 25 & 2,79 & 0,867 \\
\hline $\mathrm{G}=$ área basal; $\mathrm{S}=$ riqueza de espécies; $\mathrm{H}^{\prime}$ = índice de diversidade de
\end{tabular}

$\mathrm{G}=$ área basal; $\mathrm{S}=$ riqueza de espécies; $\mathrm{H}^{\prime}=$ índice de diversidade de Shannon; $J^{\prime}=$ índice de equabilidade de Pielou.

Embora os SAF 3 (36 espécies) e o SAF 2 (28 espécies) tenham apresentado as maiores quantidades de espécies, foram os SAF 4 e SAF 5 que apresentaram os maiores índices de diversidade e uniformidade das espécies presentes nas áreas avaliadas. Isso pode ser explicado em função das áreas dos SAF 4 e SAF 5 serem menores o que proporciona maior representatividade das diferentes espécies, em contraste aos SAFs 1, 2 e 3 que têm áreas maiores e com predominância de apenas uma ou duas espécies.

$\mathrm{Na}$ Tabela 2 encontram-se os valores dos atributos físicos do solo, que variaram consideravelmente entre os diferentes SAFs e entre as duas classes de profundidade do solo. De maneira geral, os valores obtidos para densidade de solo estão acima do valor crítico associado a este atributo, já que valores de Ds acima de $1,40 \mathrm{~kg} \mathrm{dm}^{-3}$ podem indicar compactação do solo e representar limitações à infiltração de água no solo, à aeração e ao crescimento das raízes das plantas (SILVA et al., 2015).

Com relação à macroporosidade, com exceção da camada de $0 \mathrm{~cm}$ a $20 \mathrm{~cm}$ do SAF 2, todos os SAFs apresentaram valores inferiores a $0,10 \mathrm{~m}^{3} \mathrm{~m}^{-3}$, que é considerado limitante ao crescimento radicular (REICHERT et al., 2007).

Os valores da microporosidade (Mip), por sua vez, variaram entre 0,26 e $0,44 \mathrm{~m}^{3} \mathrm{~m}^{-3}$, sendo menor nos sistemas que apresentaram solos mais densos (SAF 2 e SAF 3), devido a correlação negativa da Ds com a Mip (VIANA et al., 2011). Além disso, por consequência dos baixos valores de Map e Mip, os SAFs apresentaram resultados para o atributo porosidade total $(\mathrm{Pt})$ abaixo do valor crítico de $0,50 \mathrm{~m}^{3} \mathrm{~m}^{-3}$ (KIEHL, 1979).

Assim como os atributos físicos do solo, os valores médios referentes aos teores de matéria orgânica do solo (MOS) e teores de carbono orgânico do solo (COS) variaram consideravelmente entre os diferentes SAFs e entre as duas profundidades (Tabela 3).

Os valores de COS e MOS na camada de $0 \mathrm{~cm}$ a $20 \mathrm{~cm}$ seguiram a seguinte ordem: SAF4 $>$ SAF5 $>$ SAF1 $>$ SAF2 $>$ SAF3; enquanto que para a camada de $20 \mathrm{~cm}$ a $40 \mathrm{~cm}$ seguiram a seguinte ordem: SAF4 $>$ SAF1 $>$ SAF2 $>$ SAF3 $>$ SAF5.

A matriz de correlação (Tabela 4) evidencia algumas associações entre os atributos físicos do solo, os estoques de MOS e COS e os atributos florísticos e fitossociológicos, demostrando que os diferentes elementos dos sistemas agroflorestais interagem e possivelmente influenciam na produtividade e na sustentabilidade desses agroecossistemas. 
Tabela 2. Médias de densidade do solo (Ds), macroporosidade (Map), microporosidade (Mip) e porosidade total (Pt) em diferentes sistemas agroflorestais da Região Central de Rondônia.

Table 2. Soil bulk density (Ds), macroporosity (Map), microporosity (Mip) and total porosity (Pt) averages in different agroforestry systems of the Central Region of Rondonia.

\begin{tabular}{|c|c|c|c|c|c|c|c|}
\hline \multirow{2}{*}{$\begin{array}{l}\text { Atributos } \\
\text { Físicos }\end{array}$} & \multirow{2}{*}{$\begin{array}{l}\text { Profundidade } \\
(\mathrm{cm})\end{array}$} & \multicolumn{5}{|c|}{ Agroecossistemas } & \multirow{2}{*}{$\begin{array}{l}\mathrm{CV} \\
(\%)\end{array}$} \\
\hline & & SAF 1 & SAF 2 & SAF 3 & SAF 4 & SAF 5 & \\
\hline Ds & $0-20$ & 1,51 & 1,45 & 1,59 & 1,53 & 1,47 & 3,83 \\
\hline$\left(\mathrm{kg} \mathrm{dm}^{-3}\right)$ & $20-40$ & 1,44 & 1,48 & 1,68 & 1,43 & 1,37 & 7,88 \\
\hline Map & $0-20$ & 0,05 & 0,16 & 0,09 & 0,04 & 0,09 & 53,21 \\
\hline$\left(\mathrm{m}^{3} \mathrm{~m}^{-3}\right)$ & $20-40$ & 0,05 & 0,09 & 0,05 & 0,06 & 0,05 & 30,14 \\
\hline Mip & $0-20$ & 0,37 & 0,27 & 0,26 & 0,39 & 0,40 & 20,23 \\
\hline$\left(\mathrm{m}^{3} \mathrm{~m}^{-3}\right)$ & $20-40$ & 0,39 & 0,31 & 0,28 & 0,39 & 0,44 & 18,20 \\
\hline $\mathrm{Pt}$ & $0-20$ & 0,42 & 0,43 & 0,35 & 0,44 & 0,49 & 11,48 \\
\hline$\left(\mathrm{m}^{3} \mathrm{~m}^{-3}\right)$ & $20-40$ & 0,44 & 0,41 & 0,33 & 0,45 & 0,48 & 13,42 \\
\hline
\end{tabular}

$\overline{\mathrm{SAF}}=$ sistema agroflorestal; $\mathrm{Ds}$ = densidade do solo; Map = macroporosidade; Mip = microporosidade; $\mathrm{Pt}$ = porosidade total e CV = coeficiente de variação.

Tabela 3. Valores de matéria orgânica e carbono orgânico do solo nos diferentes sistemas agroflorestais da Região Central do Estado de Rondônia.

Table 3. Organic matter and soil organic carbon values for different agroforestry systems from the Central Region of Rondonia.

\begin{tabular}{cccccccc}
\hline \multirow{2}{*}{$\begin{array}{c}\text { Parâmetros } \\
\left(\mathrm{Mg} \mathrm{ha}^{-1}\right)\end{array}$} & \multirow{2}{*}{ Profundidade $(\mathrm{cm})$} & SAF 1 & SAF 2 & SAF 3 & SAF 4 & SAF 5 & CV $(\%)$ \\
\cline { 3 - 6 } MOS & $0-20$ & 24,48 & 19,41 & 12,00 & 31,16 & 27,99 & 32,79 \\
& $20-40$ & 17,41 & 16,32 & 13,58 & 19,23 & 11,97 & 18,62 \\
& $0-40$ & 41,89 & 35,73 & 25,58 & 50,39 & 39,96 & 23,45 \\
\hline \multirow{2}{*}{ Est COS } & $0-20$ & 42,88 & 32,50 & 21,67 & 55,21 & 47,82 & 32,90 \\
& $20-40$ & 28,67 & 28,04 & 26,25 & 32,09 & 19,10 & 17,93 \\
& $0-40$ & 71,55 & 60,54 & 47,91 & 87,30 & 66,92 & 21,66 \\
\hline
\end{tabular}

MOS = Matéria orgânica do solo; Est COS = Estoque de carbono orgânico do solo.

Tabela 4. Matriz de correlação de Pearson dos atributos florísticos, atributos físicos do solo e estoques de matéria orgânica e carbono orgânico do solo.

Table 4. Pearson correlation matrix of floristic attributes, soil physical attributes, organic matter and soil organic carbon stocks.

\begin{tabular}{|c|c|c|c|c|c|c|c|c|c|c|}
\hline & $\begin{array}{c}\mathrm{G} \\
\left(\mathrm{m}^{2} \mathrm{ha}^{-1}\right)\end{array}$ & $\begin{array}{c}\mathrm{S} \\
\text { (espécies) }\end{array}$ & $\mathrm{H}^{\prime}$ & $\mathrm{J}^{\prime}$ & $\begin{array}{c}\text { Ds } 0-20 \mathrm{~cm} \\
\left(\mathrm{~kg} \mathrm{dm}^{-3}\right)\end{array}$ & $\begin{array}{l}\text { Ds 20-40 } \\
\left(\mathrm{kg} \mathrm{dm}^{-3}\right)\end{array}$ & $\begin{array}{c}\text { Map 0-40 } \\
\left(\mathrm{m}^{3} \mathrm{~m}^{-3}\right)\end{array}$ & $\begin{array}{c}\text { Mip 0-40 } \\
\left(\mathrm{m}^{3} \mathrm{~m}^{-3}\right)\end{array}$ & $\begin{array}{l}\text { Pt } 0-40 \\
\left(\mathrm{~m}^{3} \mathrm{~m}^{-3}\right)\end{array}$ & $\begin{array}{r}\text { MOS 0-40 } \\
\left(\mathrm{Mg} \mathrm{ha}^{-1}\right) \\
\end{array}$ \\
\hline $\mathrm{G}\left(\mathrm{m}^{2} \mathrm{ha}^{-1}\right)$ & 1 & & & & & & & & & \\
\hline S (espécies) & $-0,4193^{\mathrm{ns}}$ & 1 & & & & & & & & \\
\hline $\mathrm{H}^{\prime}$ & $0,1010^{\mathrm{ns}}$ & $-0,0653^{\text {ns }}$ & 1 & & & & & & & \\
\hline $\mathrm{J}$ & $0,1380^{\mathrm{ns}}$ & $-0,1567^{\mathrm{ns}}$ & $0,9957^{*}$ & 1 & & & & & & \\
\hline Ds $0-20 \mathrm{~cm}\left(\mathrm{~kg} \mathrm{dm}^{-3}\right)$ & $0,5029^{\text {ns }}$ & $0,4172^{\mathrm{ns}}$ & $0,0132^{\mathrm{ns}}$ & $-0,0181^{\mathrm{ns}}$ & 1 & & & & & \\
\hline Ds $20-40 \mathrm{~cm}\left(\mathrm{~kg} \mathrm{dm}^{-3}\right)$ & $0,0127^{\mathrm{ns}}$ & $0,7593 *$ & $-0,4929^{\text {ns }}$ & $-0,5542^{\text {ns }}$ & $0,7300^{*}$ & 1 & & & & \\
\hline Map $0-40 \mathrm{~cm}\left(\mathrm{~m}^{3} \mathrm{~m}^{-3}\right)$ & $-0,8828^{*}$ & $0,4105^{\mathrm{ns}}$ & $-0,4619^{\text {ns }}$ & $-0,4986^{\text {ns }}$ & $-0,5513^{\text {ns }}$ & $0,1325^{\mathrm{ns}}$ & 1 & & & \\
\hline Mip $0-40 \mathrm{~cm}\left(\mathrm{~m}^{3} \mathrm{~m}^{-3}\right)$ & $0,4018^{\mathrm{ns}}$ & $-0,7149^{*}$ & $0,7163^{*}$ & $0,7719^{*}$ & $-0,3250^{\text {ns }}$ & $-0,8652 *$ & $-0,5767^{\mathrm{ns}}$ & 1 & & \\
\hline Pt $0-40 \mathrm{~cm}\left(\mathrm{~m}^{3} \mathrm{~m}^{-3}\right)$ & $-0,0490^{\mathrm{ns}}$ & $-0,6257^{\mathrm{ns}}$ & $0,6284^{\mathrm{ns}}$ & $0,6749^{\text {ns }}$ & $-0,6998^{*}$ & $-0,9778^{*}$ & $-0,1271^{\mathrm{ns}}$ & $0,8814 *$ & 1 & \\
\hline $\operatorname{MOS} 0-40 \mathrm{~cm} \quad\left(\mathrm{Mg} \mathrm{ha}^{-1}\right)$ & $0,0899^{\mathrm{ns}}$ & $-0,7524^{*}$ & $0,5354^{\mathrm{ns}}$ & $0,6044^{\mathrm{ns}}$ & $-0,3358^{\text {ns }}$ & $-0,8189^{*}$ & $-0,3990^{\text {ns }}$ & $0,7850^{*}$ & $0,7608^{*}$ & 1 \\
\hline $\operatorname{COS} 0-40 \mathrm{~cm}\left(\mathrm{Mg} \mathrm{ha}^{-1}\right)$ & $0,1302^{\mathrm{ns}}$ & $-0,7125^{*}$ & $0,5436^{\mathrm{ns}}$ & $0,6103^{\text {ns }}$ & $-0,2190^{\mathrm{ns}}$ & $-0,7422^{*}$ & $-0,4630^{\text {ns }}$ & $0,7480^{*}$ & $0,6839^{\text {ns }}$ & $0,9919 *$ \\
\hline
\end{tabular}

$\mathrm{G}$ = área basal por ha; $\mathrm{S}=$ riqueza de espécies; $\mathrm{H}^{\prime}$ = Índice de diversidade de Shannon; $\mathrm{J}^{\prime}$ = Índice de equabilidade de Pielou; Ds 0 $\mathrm{cm}$ a $20 \mathrm{~cm}=$ densidade do solo na camada de $0 \mathrm{~cm}$ a $20 \mathrm{~cm}$; Ds $20 \mathrm{~cm}$ a $40 \mathrm{~cm}=$ densidade do solo na camada de $20 \mathrm{~cm}$ a $40 \mathrm{~cm}$; Map $0 \mathrm{~cm}$ a $40 \mathrm{~cm}=$ macroporosidade na camada de $0 \mathrm{~cm}$ a $40 \mathrm{~cm}$; Mip $0 \mathrm{~cm}$ a $40 \mathrm{~cm}=$ microporosidade na camada de $0 \mathrm{~cm}$ a $40 \mathrm{~cm} ;$ Pt $0 \mathrm{~cm}$ a $40 \mathrm{~cm}=$ porosidade total na camada de $0 \mathrm{~cm}$ a $40 \mathrm{~cm} ;$ MOS $0 \mathrm{~cm}$ a $40 \mathrm{~cm}=$ matéria orgânica do solo na camada de $0 \mathrm{~cm}$ a $40 \mathrm{~cm} ; \operatorname{COS} 0 \mathrm{~cm}$ a $40 \mathrm{~cm}=$ carbono orgânico do solo na camada de $0 \mathrm{~cm}$ a $40 \mathrm{~cm} ;{ }^{\text {ns }}=$ não significativo e $*=$ significativo pela correlação de Pearson $(\mathrm{p}>0,10)$

\section{DISCUSSÃO}

Os SAF 1 e SAF 2 apresentaram os menores valores de densidade do solo (Ds) na camada de $0 \mathrm{~cm}$ a $20 \mathrm{~cm}$, provavelmente devido ao significativo aporte de matéria orgânica promovido pelos cupuaçuzeiros e seringueiras, além da intensa atividade da macro e mesofauna observado em campo.

Destaca-se que embora o SAF 3 possua composição florística e arranjo similar aos dos SAF 1 e SAF 2, observouse os maiores valores de Ds para as profundidades de $0 \mathrm{~cm}$ a $20 \mathrm{~cm}$ e $20 \mathrm{~cm}$ a $40 \mathrm{~cm}$. A provável explicação desse resultado está associada à textura arenosa do solo neste agroecossistema, tendo em vista que solos arenosos frequentemente exibem valores entre 1,35 a $1,85 \mathrm{~kg} \mathrm{dm}^{-3}$, em contrapartida de valores entre 0,95 a $1,25 \mathrm{~kg} \mathrm{dm}^{-3}$ para solos argilosos (ARAÚJO et al., 2004). Entretanto, nos demais SAFs estudados, os valores de densidade do solo encontrados foram superiores a $1,35 \mathrm{~kg} \mathrm{dm}$ ${ }^{3}$, indicando que outros fatores, além da textura do solo, podem ter influenciando no aumento da Ds.

Sendo assim, supõe-se que os altos valores de densidade do solo encontrados neste trabalho sejam consequência do pouco revolvimento do solo, da baixa densidade de plantas rasteiras e das atividades de limpeza mecanizada das áreas.

Em face deste cenário, Silva et al. (2008) colaboram para o entendimento desse raciocínio, ao mencionarem que os sistemas agroflorestais, assim como outros sistemas 
conservacionistas que realizam pouco revolvimento do solo, podem favorecer $o$ aumento da densidade do solo, macroporosidade e porosidade total na camada superficial. Adicionalmente, valores similares para densidade do solo, variando entre 1,05 a $1,65 \mathrm{~kg} \mathrm{dm}^{-3}$, foram encontrados em outros estudos (SILVA et al., 2011b; PEZARICO et al., 2013; ARÉVALO-GARDINI, 2015), demonstrando que este é um fenômeno frequentemente observado entre os diferentes tipos de SAFs. Mascarenhas et al. (2017), por exemplo, encontraram em sistema agroflorestal multiestratificado na região de Ouro Preto do Oeste, Rondônia, valores de 1,63 e 1,72 $\mathrm{kg} \mathrm{dm}^{-3}$ nas camadas de $0 \mathrm{~cm}$ a $20 \mathrm{~cm}$ e $20 \mathrm{~cm}$ a $40 \mathrm{~cm}$, respectivamente.

Os valores médios da macroporosidade variaram consideravelmente entre os diferentes SAFs, como visto anteriormente na Tabela 2, e entre as duas profundidades do solo estudadas. Os resultados abaixo de $0,10 \mathrm{~m}^{3} \mathrm{~m}^{-3}$ podem indicar limitação ao crescimento radicular das plantas nestes agroecossistemas, visto que a macroporosidade é responsável por determinar a aeração e a drenagem da água no solo (REICHERT et al., 2007). Kiehl (1979) afirma que solos adequados para desenvolvimento das atividades agrícolas expressam valores em torno de $0,17 \mathrm{~m}^{3} \mathrm{~m}^{-3}$ de macroporos e, nesse mesmo sentido, Van Lier (2001) menciona que em climas tropicais, culturas com sistema radicular profundo e com alto consumo de $\mathrm{O}_{2}$, a macroporosidade mínima deve ser em torno de $0,20 \mathrm{~m}^{3} \mathrm{~m}^{-3}$. Os baixos valores encontrados neste estudo, que diferem significantemente dos parâmetros supracitados, podem ser justificados pelo comportamento de correlação negativa entre macroporosidade e densidade do solo (RICHART et al., 2005).

Para a microporosidade (Mip), os SAFs 2 e 3 apresentaram os menores valores para as duas classes de profundidade, sendo que este resultado pode ser explicado pela textura mais arenosa destes agroecossistemas, visto que solos arenosos geralmente apresentam menores valores para microporosidade do que solos argilosos (KLEIN, 2005). De forma semelhante e corroborando com a explicação acima, Mascarenhas et al. (2017) ao avaliarem os atributos físicos do solo entre diferentes sistemas de cultivo, inclusive um sistema agroflorestal, demonstraram que em função do maior percentual de areia na granulometria do solo, obteve-se menores valores para microporosidade.

Além disso, é importante destacar que a microporosidade do solo é influenciada, principalmente, pelo material de origem e pelos processos de formação do solo, entretanto, o aumento da densidade do solo, e o consequente aumento da compactação do solo, pode reduzir a proporção de macroporos e elevar a de microporos (VIANA et al., 2011), o que poderia explicar os maiores valores deste atributo nos SAF 1, SAF 4 e SAF 5.

Para a porosidade total do solo, os valores encontrados foram abaixo do valor crítico de $0,50 \mathrm{~m}^{3} \mathrm{~m}^{-3}$ estabelecido por Kiehl (1979). No SAF 3 verificou-se os menores valores para as duas profundidades, como consequência dos menores valores de microporosidade e maiores valores de densidade do solo. Em contraste, o SAF 5 apresentou os maiores valores para este atributo, em decorrência dos maiores valores de microporosidade e menores valores de densidade do solo. Assim como para outros atributos físicos do solo, as variações verificadas para Pt podem ser associadas à Ds, visto que este é o fator que mais influência no arranjo das partículas estruturais do solo.
Embora, de modo geral, os resultados obtidos para os atributos físicos do solo (Ds, Map, Mip e Pt) não estão de acordo com os parâmetros considerados ideais para crescimento e desenvolvimento vegetal de sistemas de cultivo convencionais, as plantas observadas em campo estão crescendo e produzindo normalmente. Portanto, supõe-se que fatores como a matéria orgânica do solo, a atividade da macro e mesofauna e adaptações do sistema radicular possam mitigar os efeitos dos altos valores de densidade e baixos valores de porosidade. Como consequência, talvez os valores críticos utilizados não são adequados para expressar a qualidade do solo em sistemas agroflorestais. Conforme indicado anteriormente, os atributos são influenciados diretamente pelos diferentes sistemas de manejo do solo e caracterizam as condições em que as plantas se desenvolvem, apontando ou não sobre a necessidade de novas técnicas de cultivo (SILVA et al., 2015).

Já em relação aos resultados de matéria orgânica do solo (MOS) e carbono orgânico do solo (COS), os altos valores para os SAF 4 e SAF 5 provavelmente estão associados à grande cobertura de plantas e ao intenso aporte de material orgânico observado em campo, já que estes sistemas são compostos, majoritariamente, por plantas de café de alta produtividade que depositam grande quantidade de material vegetal na superfície do solo. Também avaliando o COS em café agroflorestal, Vilela; Mendonça (2013) encontraram valor de 73,26 Mg ha' para a profundidade de $0 \mathrm{~cm}$ a $40 \mathrm{~cm}$, consideravelmente similar aos do SAF $4\left(87,30 \mathrm{Mg} \mathrm{ha}^{-1}\right)$ e do SAF $5(66,92 \mathrm{Mg}$ $\left.\mathrm{ha}^{-1}\right)$. Os autores destacam que a implantação do café no sistema agroflorestal, que antes era usada para o cultivo de arroz e feijão, aumentou os estoques de $\mathrm{C}$ em virtude das mudanças de manejo.

De modo geral, os valores de COS e MOS para a camada superior $(0 \mathrm{~cm}$ a $20 \mathrm{~cm})$ foram maiores do que para a camada inferior $(20 \mathrm{~cm}$ a $40 \mathrm{~cm})$, seguindo a tendência normal do perfil do solo de concentrar maior quantidade de carbono na superfície, devido a deposição e decomposição do material vegetal. Observa-se ainda que na profundidade de $20 \mathrm{~cm}$ a $40 \mathrm{~cm}$ a variação entre as médias (coeficiente de variação) foi menor para os dois atributos, o que poderia indicar que camadas mais profundas são menos influenciadas pelo manejo aplicado.

Em sistemas agroflorestais, assim como em outros sistemas conservacionistas, realiza-se pouco revolvimento e incorporação de matéria orgânica em camadas mais profundas do solo, o que acaba favorecendo a permanência do carbono no solo, principalmente na camada superficial, pois os resíduos vegetais podem ficar estocados por longo período de tempo. Além disso, outro fator que influencia diretamente a quantidade e permanência de $\mathrm{C}$ no solo é o adensamento de árvores, já que arranjos mais densos possuem mais plantas e produzem maior quantidade de material vegetal, além de apresentar copas fechadas que dificultam a entrada de raios solares e retardam a decomposição dos resíduos vegetais. Em contraste, arranjos menos densos possuem menor cobertura e facilitam a entrada de raios solares que aceleram a decomposição do carbono residual.

A comparação com outros estudos sobre carbono em sistemas agroflorestais evidencia grande heterogeneidade dos resultados. Valores de COS encontrados em sistema agroflorestal multiestratificado no município de Ouro Preto do Oeste (RO) foram consideravelmente similares: 24,79 $\mathrm{Mg} \mathrm{ha}^{-}$ ${ }^{1}$ para a camada de $0 \mathrm{~cm}$ a $20 \mathrm{~cm}$ e $17,26 \mathrm{Mg} \mathrm{ha}^{-1}$ para a camada 
de $20 \mathrm{~cm}$ a $40 \mathrm{~cm}$ (MASCARENHAS et al., 2017). Contudo outros estudos evidenciam maiores variações, como o estudo de Rocha et al. (2014) que encontraram valores variando entre 29,40 e 74,14 $\mathrm{Mg} \mathrm{ha}^{-1}$ para agroflorestas no município de Grão Mogol (MG). Esses diferentes resultados vão de encontro à ideia de que os teores e estoques de matéria e carbono orgânico do solo dependem de diversos fatores como uso e manejo do solo, classificação do solo, profundidade, clima e bioma (PARRON et al., 2015).

$\mathrm{Na}$ matriz de correlação (Tabela 4) relevam-se algumas associações entre os atributos físicos do solo, os estoques de MOS e COS e os atributos florísticos e fitossociológicos dos sistemas agroflorestais. A densidade do solo (Ds) na camada de $20 \mathrm{~cm}$ a $40 \mathrm{~cm}$ apresentou correlação significativa positiva com a riqueza de espécies (S) e com a Ds na camada de $0 \mathrm{~cm}$ a $20 \mathrm{~cm}$; e correlação negativa com microporosidade (Mip), porosidade total $(\mathrm{Pt})$ e estoques de MOS e COS. Supõe-se que a correlação da $\mathrm{S}$ com a Ds esteja aliada a maior diversidade de sistemas radiculares que ocupam maior quantidade de poros e, por isso, aumentam a densidade do solo. A correlação positiva da Ds na camada de $0 \mathrm{~cm}$ a $20 \mathrm{~cm}$ com a Ds na camada de $20 \mathrm{~cm}$ a $40 \mathrm{~cm}$ é de certa forma esperada, pois altos valores de densidade na camada superior pressupõem altos valores na camada inferior.

Considera-se que a correlação negativa forte $(>0,80)$ da Ds com Mip e Pt esteja associada à compactação do solo, que diminui a quantidade de poros. Valores de densidade do solo com correlação inversa ao de porosidade total têm sido relatados em outros estudos envolvendo sistemas agroflorestais (NEVES et al., 2007). A densidade do solo é o atributo que pode impor limitações ao crescimento radicular devido sua influência no espaço poroso, na umidade e na resistência do solo à penetração (SOUZA NETO et al., 2008). Como consequência, esta compactação prejudica o crescimento das raízes e a consequente produção vegetal das plantas, o que poderia explicar a correlação negativa de força mediana da Ds na camada de $20 \mathrm{~cm}$ a $40 \mathrm{~cm}$ com os valores de MOS e COS.

Além da densidade do solo, a porosidade total também apresentou associação com a MOS. A correlação de força mediana da Pt com MOS pode sugerir que solos com melhor estrutura influenciam positivamente na produção vegetal. Além disso, a microporosidade mostrou correlação positiva com o índice de diversidade de Shannon (H') e o índice de equabilidade de Pielou (J). Embora a Mip do solo seja determinada principalmente pelo material de origem e pelos processos de formação do solo (VIANA et al., 2011), possivelmente a maior diversidade e uniformidade das espécies e seus sistemas radiculares possam estar elevando a quantidade de microporos do solo, contribuindo positivamente para a estrutura do solo. Por outro lado, a Map revelou correlação negativa com área basal $(\mathrm{G})$, indicando que a arranjos mais densos diminuem a quantidade de macroporos, o que poderia comprometer a areação do solo e o crescimento das raízes.

A correlação negativa da MOS e COS com a riqueza de espécies possibilita a suposição de que a maior quantidade de espécies não propicia necessariamente maior quantidade de MOS e COS. Provavelmente a quantidade de material vegetal (ou seja, arranjos mais densos) depositado no solo seja mais importante para o estoque de carbono no solo do que a diversidade da composição florística. Esta suposição é reforçada pelo fato de que a diversidade não apresentou correlação com MOS e COS. Além disso, é necessário ressaltar que se os arbustos de café nos SAFs 4 e 5 tivessem sido mensurados, os valores de área basal $(\mathrm{G})$ seriam maiores e a correlação com MOS e COS provavelmente seria significativa, corroborando para o argumento de que quantidade de material é mais importante do que sua diversidade.

Por último, a correlação positiva da MOS e COS com Mip e Pt permite supor que a maior quantidade de poros no solo, principalmente de microporos que são responsáveis por reter e disponibilizar água para as plantas (REICHERT et al., 2007), proporciona melhoria do ambiente para crescimento das raízes, desenvolvimento das plantas e produção vegetal.

A partir da análise dos resultados obtidos, é possível inferir que sistemas com maior densidade de plantas, com maior complexidade estrutural e com melhores condições de estrutura do solo, favorecem a produtividade vegetal e os estoques de matéria orgânica e carbono orgânico no solo. Os SAF 4 e SAF 5, por exemplo, que apresentaram maior diversidade e maior cobertura do solo pelo cafeeiro, demonstraram os maiores valores de MOS e COS. Acredita-se que determinadas práticas de manejo, tais como estruturação vertical, maior adensamento de plantas, uso de plantas rasteiras de cobertura e limpeza manual da área, podem contribuir para a melhoria dos atributos edáficos e acúmulo de carbono em sistemas agroflorestais.

\section{CONCLUSÕES}

Os atributos florísticos e fitossociológicos variaram consideravelmente em função das diferenças de composição florística e estrutura dos sistemas agroflorestais.

Os atributos físicos do solo não estão nos padrões considerados ideais pela literatura específica, contudo as plantas estão crescendo e produzindo normalmente, sugerindo que talvez esses parâmetros não são adequados para todos sistemas de uso da terra.

Técnicas de manejo como arranjo mais adensado, uso de plantas de cobertura e limpeza manual da área, podem possibilitar a melhoria da qualidade do solo e o sequestro de carbono em sistemas agroflorestais.

\section{REFERENNCIAS}

ABNT - ASSOCIAÇÃO BRASILEIRA DE NORMAS TÉCNICAS. NBR 6484: Sondagens de simples reconhecimento com SPT - Método de ensaio. Rio de Janeiro: ABNT, 2001. 17p.

ALTIERI, M. Agroecologia: bases científicas para uma agricultura sustentável. 3. ed. Rio de Janeiro: Editora Expressão Popular, 2012. 400 p.

ALVARES, C. A.; STAPE, J. L; SENTELHA, P. C.; GONÇALVES, J. L. M.; SPAROVEK, G. Köppen's climate classification map for Brazil. Meteorologische Zeitschrift, Berlin, v. 22, n. 6, p. 711-728, 2013. DOI: https://dx.doi.org/10.1127/0941-2948/2013/0507

ARAÚJO, M. A.; TORMENA, C. A.; SIlVA, A. P. Propriedades físicas de um Latossolo Vermelho distrófico cultivado e sob mata nativa. Revista Brasileira de Ciência do Solo, Viçosa, n. 28, p. 337-345, 2004. DOI: https://dx.doi.org/10.1590/s0100-06832004000200012

ARÉVALO-GARDINI, E.; CANTO, M.; ALEGRE, J.; LOLI, O.; JULCA, A.; BALIGAR, V. Changes in Soil Physical and Chemical Properties in Long Lerm Improved Natural and Traditional Agroforestry Management Systems of 
Cacao Genotypes in Peruvian Amazon. PLoS ONE, San Francisco, v. 10, n. 7, 2015. DOI: https://dx.doi.org/10.1371/journal.pone.0132147

CARR, D. L.; LOPEZ, A. C.; BILSBORROW, R. E. The population, agriculture, and environment nexus in Latin America: country-level evidence from the latter half of the twentieth century. Population and Environment, New York, v. 30, n. 6, p. 222-246, 2009. DOI: https://doi.org/10.1007/s11111-009-0090-4

DONAGEMA, G. K.; CAMPOS, D. V. B. CALDERANO, W. G. TEIXEIRA W. G.; VIANA, J. H. m. (Org.). Manual de métodos de análise de solos. 2. ed. Rio de Janeiro: Embrapa Solos, 2011.230 p.

FEARNSIDE, P. M.; GRAÇA, P. M. L. A. BR-319: A rodovia Manaus-Porto Velho e o impacto potencial de conectar o arco de desmatamento à Amazônia central. Novos Cadernos NAEA, Belém, v. 12, n. 1, p. 19-50, 2009. DOI: https://dx.doi.org/10.5801/ncn.v12i1.241

GAMA-RODRIGUES, E. F.; BARROS, N. F.; VIANA, A P.;SANTOS, G. A. Alterações na biomassa e na atividade microbiana da serapilheira e do solo, em decorrência da substituição de cobertura florestal nativa por plantações de eucalipto, em diferentes sítios da região sudeste do Brasil. Revista Brasileira de Ciência do Solo, Viçosa, v. 32, n. 4, p. 1489-1499, 2008. DOI: https://dx.doi.org/10.1590/s0100-06832008000400013

KIEHL, E. J. Manual de edafologia. São Paulo: Agronômica Ceres, 1979. 264 p.

KLEIN, V. A. Propriedades do solo e manejo da água em ambientes protegidos com cultivo de morangueiro e figueira. Passo Fundo, RS: Editora UPF, 2005. 61 p.

LIMA, S. S.; LEITE, L. F. C.; OLIVEIRA, F. C.; COSTA, D. B. Atributos químicos e estoques de carbono e nitrogênio em argissolo vermelho-amarelo sob sistemas agroflorestais e agricultura de corte e queima no norte do Piauí. Revista Árvore, Viçosa, v. 35, n. 1, p. 51-60, 2011. DOI: 67622011000100006

MALHI, Y.; ROBERTS, J. T.; BETTS, R. A.; KILLEEN, T. J.; LI, W.; NOBRE, C. A. Climate change, deforestation and the fate of the Amazon. Science, Washington, v. 319, n. 5860, p. 169-172, 2008. DOI: https://dx.doi.org/10.1126/science.1146961

MASCARENHAS, A. R. P.; SCCOTI, M. S. V.; MELO, R. R.; CORRÊA, F. L. O.; MAIA, E.; ANDRADE, R. A.; BERGAMIN, A. C.; MULLER, M. W. Atributos físicos e estoques de carbono do solo sob diferentes usos da terra em Rondônia, Amazônia Sul-Ocidental. Pesquisa Florestal Brasileira, Colombo, v. 37, n. 89, p.19-27, 2017.

https://dx.doi.org/10.4336/2017.pfb.37.89.1295

NEVES, C. M. N.; SILVA, M. L. N.; CURI, N.; CARDOSO, E. L.; MACEDO, R. L. G.; FERREIRA, M. M.; SOUZA, F. S. Atributos indicadores da qualidade do solo em sistema agrossilvopastoril no noroeste do estado de Minas Gerais. Scientia Forestalis, Piracicaba, n. 74, p. 45-53, 2007.

PARRON, L. M.; RACHWAL, M. F. G.; MAIA, C. M. B. de F. Estoques de carbono no solo como indicador de serviços ambientais. In: PARRON, L. M.; GARCIA, J. R.; OLIVEIRA, E. B. de; BROWN, G. G; PRADO, R. B. (Ed.). Serviços ambientais em sistemas agrícola e florestais do Bioma Mata Atlântica. Brasília: Embrapa, 2015, p. 92-100.

PEZARICO, C. R.; VITORINO, A. C. T.; MARCANTE, F. M.; DANIEL, O. Indicadores de qualidade do solo em sistemas agroflorestais. Revista de Ciências Agrárias, Belém, v. 56, n. 1, p. 40-47, 2013. DOI: http://dx.doi.org/10.4322/rca.2013.004

REICHERT, J. M.; SUZUKI, L. E. A. S.; REINERT, D. J. Compactação do solo em sistemas agropecuários e florestais: Identificação, Efeitos, Limites Críticos e Mitigação. Tópicos em Ciência do Solo, v. 5, p. 49-134, 2007.

RICHART, A.; TAVARES FILHO, J.; BRITO, O. R.; LLANILLO, R. F.; FERREIRA, R. Compactação do solo: causas e efeitos. Semina: Ciências Agrárias, Londrina, v. 26, n. 3, p. 321-344, 2005. DOI: https://dx.doi.org/10.5433/1679-0359.2005v26n3p321

ROCHA, G. P.; FERNANDES, L. A.; CABACINHA, C. D.; LOPES, I. D. P.; RIBEIRO, J. M.; FRAZÃO, L. A.; SAMPAIO, R. A. Caracterização e estoques de carbono de sistemas agroflorestais no Cerrado de Minas Gerais. Ciência Rural, Santa Maria, v. 44, n. 7, p. 1197-1203, 2014. DOI: https://dx.doi.org/10.1590/0103$8478 \mathrm{cr} 20130804$

RODRIGUES, M.J.M.; SILVA, L.M.; MARCHÃO, R.L. SOUZA, A.M.; WADT, P.G.S.; OLIVEIRA, L.C. Espectroscopia no infravermelho próximo para a quantificação de carbono em solos da bacia do Acre. Biota Amazônia, Macapá, v. 6, n. 1, p. 119-124, 2016. DOI: http://dx.doi.org/10.18561/2179-

5746/biotaamazonia.v6n1p119-124

SANTOS, H. G. dos; JACOMINE, P. K. T.; ANJOS, L. H. C. dos; OLIVERA, V. A. de; LUMBRERAS, J. F.; COELHO, M. R.; ALMEIDA, J. A. de; ARAÚJO FILHO, J. C. de; OLIVEIRA, J. B. de; CUNHA, T. J. F. Sistema brasileiro de classificação de solos. 3 . ed. Brasília: Embrapa, 2013. 353 p.

SILVA, R. F.; BORGES, C. D.; GARIB, D. M.; MARCANTE, F. M. Atributos físicos e teor de matéria orgânica na camada superficial de um Argissolo vermelho cultivado com mandioca sob diferentes manejos. Revista Brasileira de Ciência do Solo, Viçosa, v. 32, n. 6, p. 2435 2441, 2008. DOI: https://dx.doi.org/10.1590/s010006832008000600021

SILVA, R. C. S.; ALMEIDA, J. C. R.; BATISTA, G. T.; FORTES NETO, P. Os indicadores físicos, químicos e biológicos da qualidade do solo e da sustentabilidade dos ambientes naturais. Repositório Eletrônico Ciências Agrárias, Coleção Ciências Ambientais, p. 1-13, 2011 a. SILVA, D. C.; SILVA, M. L. N.; CURI, N.; OLIVEIRA, A. H.; SOUZA, F. S.; MARTINS, S. G.; MACEDO, R. L. G. Atributos do solo em sistemas agroflorestais, cultivo convencional e floresta nativa. Revista de Estudos Ambientais, Blumenau, v. 13, n. 1, p. 77-86, 2011b. DOI: http://dx.doi.org/10.7867/1983-1501.2011v13n1p77-86

SILVA, H.; FAVARETTO, N.; CAVALIERI, K. M. V.; DIECKOW, J.; VEZZANI, F. M.; PARRON, L. M.; CHEROBIM, V. F.; MARIOTI, J.; FERRARI NETO, H. Atributos físicos do solo e escoamento superficial como indicadores de serviços ambientais. In: Parron, L. M. et al. In: PARRON, L. M.; GARCIA, J. R.; OLIVEIRA, E. B. de; BROWN, G. G.; PRADO, R. B. (Ed.). Serviços 
Lenci et al.

ambientais em sistemas agrícola e florestais do Bioma Mata Atlântica. Brasília: Embrapa, 2015. p. 71-83.

SOUZA NETO, E. L.; ANDRIOLI, I.; BEUTLER, A. N.; CENTURION, J. F. Atributos físicos do solo e produtividade de milho em resposta a culturas de pré safra. Pesquisa Agropecuária Brasileira, Brasília v. 43, n. 2, p. 255-260, 2008. DOI: https://dx.doi.org/10.1590/s0100204x2008000200015

VAN LIER, Q. J. Oxigenação do sistema radicular: uma abordagem física. Revista Brasileira de Ciência do Solo, Viçosa, v. 25, n. 1, p. 233-238, 2001. DOI: https://dx.doi.org/10.1590/s0100-06832001000100025
VIANA, E. T.; BATISTA, M. A.; TORMENA, C. A.; COSTA, A. C. S.; INOUE, T. T. Atributos físicos e carbono orgânico em latossolo vermelho sob diferentes sistemas de uso e manejo. Revista Brasileira de Ciência do Solo, Viçosa, v. 35, n. 1, p. 2105-2114, 2011. DOI: http://dx.doi.org/10.1590/S0100-06832011000600025

VILELA, E. F.; MENDONÇA, E. S. Impacto de sistemas agroflorestais sobre a matéria orgânica do solo: modelagem de carbono e nitrogênio. Coffee Science, Lavras, v. 8, n. 3, p. 354-363, 2013. 